\title{
Exploración del Conocimiento Sobre la Relación de Parentesco como Contenido Educativo Para un Currículum Escolar Intercultural en Comunidades Mapunche
}

\section{Exploring the Knowledge of Family Relationships as an Educational Content for an Intercultural School Curriculum in the Mapunche Communities}

\author{
Segundo Quintriqueo M. * \\ Gisèle Maheux **
}

\begin{abstract}
Resumen
Desde que las escuelas existen en comunidades mapunche, los contenidos educativos del patrimonio cultural no han sido formalizados en el currículum escolar. Un aspecto considerado importante de este patrimonio concierne a la relación de parentesco, ligado a la formación de persona en la perspectiva de la socialización mapunche y a los contenidos del Estudio del Medio Natural, Social y Cultural del currículum escolar formal. A través de una investigación cualitativa con enfoque etnográfico, el artículo explora la ausencia de estos contenidos en el currículum escolar y presenta la pregunta sobre el ángulo del proceso de escolarización histórico en contexto de escuelas situadas en comunidades mapunche. El propósito es la construcción de un marco conceptual en relación al conocimiento sobre la relación de parentesco y su proceso de socialización en el contexto de la familia y la comunidad. Los datos han sido sometidos a un análisis de contenido temático, para entregar una descripción del estado actual del conocimiento sobre la relación de parentesco, para su documentación como contenido de Formación Inicial Docente en Educación Intercultural y su incorporación formal al currículum de escuelas situadas en contextos indígenas, particularmente en comunidades mapunche. La discusión de los resultados nos evidencian que la familia y la comunidad continúan un proceso de socialización que permite transmitir ciertas categorías de contenidos educativos mapunche deseables de ser integrados para la formación de persona en el medio escolar.
\end{abstract}

Palabras claves: educación mapunche e intercultural, currículum escolar y socialización.

* Profesor de La Universidad Católica de Temuco, email: squintri@uct.cl

** Professor al l'Université du Québec en Abitibi-Temiscamingue, email: gisele.maheux@uqat.ca 


\begin{abstract}
Since the school institution has taken place in mapunche communities, the mapunche cultural patrimony has not been formalized as a content in the educational school curriculum. An aspect these patrimony components considered important are related to the study of natural, social and cultural milieu. Through an ethnographically focused qualitative research, this article explores the absence of the indigenous cultural patrimony as formal area of knowledge in the educational content of the school curriculum, and explores this question from the point of view of the historical schooling process in the context of schools located in mapunche communities. The goal of this study is to develop a conceptual representation of family relationship knowledge and the socialization process in the context of the family and the community. A thematic content analysis of data was performed in order to describe the current state of knowledge of family relationship, and then to provide relevant knowledge to document the training content of Initial Teacher Training in Intercultural Education and the curriculum of schools located in an indigenous context, particularly the mapunche communities. The discussion of the results shows that the family and the community carry on a socialization process that allows transmitting certain mapunche educational content categories which would be worth incorporating into the individual training of school students.
\end{abstract}

Key words: Mapunche and intercultural education, school curriculum, and socialization.

El artículo explora un aspecto del subsector de aprendizaje Estudio y Comprensión del Medio Natural, Social y Cultural del currículum escolar ${ }^{1}$ ofrecido a alumnos de escuelas situadas en comunidades mapunche ${ }^{2}$ de la IX Región y se inscribe en el contexto de la Formación Inicial Docente en Educación Intercultural de la Universidad Católica de Temuco. El aspecto de contenidos del currículum escolar que aborda es el conocimiento mapunche sobre la relación de parentesco.
El estudio evidencia que los conocimientos sobre la relación de parentesco, como otros aspectos del patrimonio cultural mapunche, están ausentes en el currículum escolar. Sin embargo, en el contexto de la familia mapunche existe una educación que aún está en referencia a una lógica tradicional del saber y conocimiento sobre la relación de parentesco. Cuando los niños se incorporan a la escuela, se observa que éstos se enfrentan a una escolarización realizada sobre la

1 El artículo presenta un aspecto de los resultados de la investigación "Representación de la relación de parentesco que la familia mapunche posee y su transmisión a niños mapunche de edad escolar primaria en relación con los contenidos de Formación Inicial Docente" en Educación Intercultural de la Universidad Católica de Temuco, en Chile, en el marco de la tesis de Maestría en Educación presentada "À l'Université du Québec en AbitibiTemiscamingue, au Québec-Canada", de 1999 a 2002.

2 El término "mapunche" es propio de la lengua-mapunzugun-hablada por miembros del Pueblo Mapunche y no lleva "s" en plural, siendo ésta una regla aplicable a todos los términos en mapunzugun en el texto. La " $n$ " en el término "mapunche" hace referencia al aspecto de asentamiento y a un patrimonio sociocultural propio. 
base de un currículum formal que oculta el contenido sociocultural apropiado en la socialización primaria. Este proceso desplaza la personalidad del niño a una situación de ruptura con su propia experiencia de socialización y, por lo tanto, con su propia identidad individual y social mapunche. El objetivo general del artículo es presentar la relación de parentesco como contenidos de socialización en el marco cultural y educativo de la familia mapunche, para analizar sus posibilidades de incorporación al Estudio y Comprensión del Medio Social y Cultural del currículum escolar.

Los resultados de la investigación permiten explicitar la existencia de un conjunto organizado de conocimientos y de prácticas sociales en el contexto de las familias mapunche. Ello pone igualmente en evidencia la transmisión de prácticas de socialización a las nuevas generaciones en relación con contenidos educativos y, consecuentemente, la constatación de un proceso de pérdida progresiva de la identidad cultural. Estas observaciones suscitan un cuestionamiento y un deseo en relación con la incorporación de estos contenidos educativos al currículum escolar, en referencia a los procesos de construcción de identidad de los jóvenes en las comunidades mapunche.

\section{La Escolarización en Contexto Mapunche}

Hoy día existe una educación escolar que se orienta a transmitir contenidos que se privilegian desde las sociedades occidentales para la prolongación de la sociedad y la cultura de generación en generación. La elección de estos contenidos definidos por las sociedades toman en consideración los elementos prospectivos derivados del análisis internacional de la educación. En esta perspectiva la escuela en contexto intercultural, como en otros medios, se le confiere una doble misión: a) debe representar la continuidad social, por cuanto es transmisora del legado cultural de generaciones anteriores; $y$ b) debe promover el cambio social (Díaz, 1997). Esta doble misión de la educación escolar está plasmada en los diferentes niveles de concreción del currículum escolar, donde los contenidos son organizados tanto en su dimensión técnica como práctica, para preparar a las nuevas generaciones en las competencias requeridas y establecidas en la sociedad. En esta lógica, quienes deciden en las sociedades realizan toda una elección de aquello que juzgan que es de interés de la propia sociedad, lo que hoy día incluye las presiones inherentes a la globalización de las comunicaciones y de los intercambios.

\subsection{El Caso de las Poblaciones Indígenas}

En el caso de las poblaciones indígenas, si uno se remonta a la época de la conquista, la educación se orienta a los objetivos de asimilación. En el contexto local, históricamente la escolarización de niños mapunche se ha inscrito en un proceso de contacto entre la sociedad occidental y el Pueblo Mapunche. En este proceso, según Guevara (1904), la misión de escolarización se resume en la colonización y evangelización de los miembros del Pueblo Mapunche (Noggler, 1972; Foerster, 1988). La evangelización se entiende como un proceso por el cual las nuevas generaciones mapunche se convierten a un ser cristiano y abandonan progresivamente las concepciones acerca del mundo social y natural definido en el marco de su propia cultura. Esta forma de razonamiento se relaciona con la hipótesis histórica de que la cosmovisión mapunche influye negativamente en el aprendizaje y el desarrollo de competencias en base a un conjunto de contenidos educativos consignados en el currículum escolar, como una acción tradicional del sistema educativo. Esta lógica de escolarización ignora los saberes y conocimientos de otras culturas, donde se presenta a la sociedad mapunche como una cultura inferior, mediante prejuicios y estereotipos transmitidos a través del discurso del profesor (Quilaqueo y Merino, 2003; Merino y 
Quilaqueo, 2003). En este sentido, históricamente se ha impedido la incorporación de saberes y conocimientos propios como una oportunidad para revertir la pérdida progresiva de la identidad individual y social del niño mapunche en el medio escolar y comunitario.

En el caso de las comunidades mapunche así como en otros pueblos o naciones indígenas de América del Norte y América del Sur, se observa que los conocimientos que constituyen patrimonio sociocultural no son considerados en los procesos de organización del contenido para la enseñanza y el aprendizaje a nivel de estructura del Estado en general. Según Essomba (1999), en contextos interculturales existen dos grandes principios que están en la base de los procesos pedagógicos: a) los miembros de una comunidad poseen un marco cultural propio, saberes y conocimientos que les han permitido sobrevivir en un entorno social y natural; y b) cualquier intervención educativa tiene consecuencias, casi siempre negativas, cuando no se toma en cuenta lo que allí existe. En esta perspectiva, la aspiración, percepción y concepción sobre la escuela y la educación dependen también de cada grupo social y cultural (Sarrasin, 1994; Forquin, 1997).

En el plano específico es posible señalar que las familias y las comunidades mapunche poseen representaciones diferentes respecto de la sociedad occidental en relación con la formación de persona, expresadas en sistemas de valores y saberes socioculturales. Ello se debe a que la escuela y la familia presentan finalidades e intereses educativos diferentes frente a la formación de los niños. Sin embargo, en la educación escolar desarrollada en las comunidades mapunche existe un interés cada vez mayor sobre la necesidad de mejorar la calidad de la educación, orientada a una profesión necesaria de las nuevas generaciones para enfrentar la sociedad actual.

\subsection{Las Comunidades Mapunche}

En el contexto de comunidades mapunche observamos que las motivaciones y proyectos propios de los alumnos están marcados por el recorrido de escolarización y la socialización primaria, las que al no establecer la comunicación, generan problemas de deserción escolar, abandono y analfabetismo. Por otra parte, la formación de profesores de Educación Básica, desde 1992, con un enfoque en educación intercultural y en alguna medida la Reforma Educativa desde 1996 (Quilaqueo y Quintriqueo, 2003), deberían modificar en esencia la educación escolar. Sin embargo, los alumnos de origen mapunche y de sectores pobres de la IX Región atraviesan actualmente un período difícil, donde el rendimiento escolar es uno de los más bajos del país ${ }^{3}$ en los últimos años.

En el sistema educativo escolar chileno, la educación se define como "el desarrollo de la personalidad del alumno y su capacitación para la vinculación e integración a su medio social, a través del aprendizaje de contenidos mínimos obligatorios en el proceso educativo formal. La educación formal se refiere a aquella que se entrega de manera estructurada científicamente y que se entrega de manera sistemática por establecimientos escolares" (LOCE ${ }^{4}$, Art. 7, 1990). En el caso de la socialización de los alumnos de comunidades mapunche esta forma de definir la educación y los contenidos escolares deriva en tres problemas fundamentales: 1) la educación escolar está orientada a la integración de los sujetos de la acción educativa a una sociedad y cultura única representada en el currículum escolar; 2) la enseñanza y la educación se limitan

\footnotetext{
3 El Sistema de Medición de la Calidad de la Educación (2000), señala que el puntaje promedio de alumnos de escuelas situadas en comunidades (222 puntos) está por debajo del promedio nacional (250 puntos), asociado a un mayor indice de ruralidad y pobreza, lo cual evidencia la necesidad de generar una educación diferente en contexto mapunche (www.mineduc.cl).

4 Ley Orgánica Constitucional de Enseñanza.
} 
sólo a unas finalidades y contenidos educativos que están científicamente estructurados, según la lógica de la sociedad y cultura representada en el currículum escolar; y 3) la educación escolar desarrollada en contexto de comunidades desconoce explícitamente otras categorías de contenidos que no sea la occidental, tal es la literatura oral y el conocimiento sobre la relación de parentesco en el marco de la sociedad y cultura mapunche. Esto, aunque los alumnos presentan diferencias en la lógica del saber y conocimiento educativo para la formación de persona. No obstante, hoy existe una apertura para integrar saberes y conocimientos sobre la relación de parentesco como contenidos de enseñanza escolar en el subsector Estudio y Comprensión del Medio Natural, Social y Cultural del actual currículum escolar (Planes y Programas de Estudios NB1, 1996; NB2, 1997).

El análisis planteado permite dilucidar que existe una falta de conocimientos referidos al patrimonio cultural mapunche en el currículum escolar de escuelas situadas en comunidades, para escolarizar a las nuevas generaciones en una lógica de pensamiento que responda a la comunidad y al marco curricular nacional. Todo esto en referencia al medio natural, social y cultural, lo cual permitirá a los alumnos continuar la construcción de su identidad individual y social. Esto justifica su discusión a fin de documentar la relación de parentesco como contenido educativo en el Estudio y Comprensión del Medio Social y Cultural, lo cual permita mejorar la educación y la comunicación intercultural de las nuevas generaciones mapunche con los miembros de la sociedad nacional y los actores del proceso educativo escolar.

\section{Conceptualización Sobre la Relación de Parentesco como Contenido Educativo}

El parentesco se relaciona con la organización y estructura social de las familias y las comunidades mapunche, las cuales se han conservado a pesar de los cambios sociales, políticos y territoriales (Faron, 1997; Gissi, 1997). Hoy día, las familias resultantes de las ramificaciones del tronco parental mantienen una tendencia a permanecer como grupos variables y en movimiento en el territorio de las comunidades vecinas, sobre la base de la estructura y organización de la relación de parentesco que ha dejado el ancestro común. Estos grupos residen en las comunidades y conservan una relación con las cuatro raíces del parentesco mapunche; el küpan y tuwïn (origen parental paterno), el küpan y tuwïn (origen parental materno). En este contexto, la expresión "ser mapunche" se refiere a un estado de personalidad propiamente mapunche, cuyas características se construyen sobre la base del interaccionismo social, en la relación tiempo-espacio en la comunidad y el conocimiento concerniente al tronco parental común. Las formas de pensamientos y de saberes se construyen a través de la experiencia de relación social cotidiana, formal, informal, personal y colectiva sobre la relación de parentesco, ligadas a una concepción y visión de mundo. La permanencia física de los grupos familiares en una misma comunidad está asociada a un conocimiento y valoración sobre la relación de parentesco en la memoria social mapunche. Este conocimiento constituye un patrimonio cultural que da sentido a la forma de ser mapunche, para comprender la realidad sociocultural actual (Quintriqueo et al., 1996). Se entiende por patrimonio cultural mapunche a todos aquellos saberes tecnológicos, sociales, culturales, cognitivos y educacionales propios, producto de una construcción sociohistórica del saber para comprender la realidad (Bonfil, 1993).

Desde la educación la relación de parentesco se define como un conjunto de conocimientos, de prácticas, de saberes, valores, normas y reglas representadas en la familia como categorías de contenidos para la formación de persona, a través de la socialización de la memoria social que hoy portan los kimche. La memoria social constituye un con- 
junto de saberes y conocimientos sobre la relación de parentesco representada en la familia mapunche y construida en la relación social entre los miembros del parentesco.

Aquí algunos elementos de definición de los conceptos centrales en el estudio del conocimiento sobre la relación de parentesco mapunche: la educación, la socialización, la representación social y el currículum escolar.

\subsection{Los conceptos de educación y socialización}

Los conceptos de educación y de socialización tienen su punto de encuentro en la función de transmitir saberes y conocimientos a las nuevas generaciones.

\subsubsection{LA EDUCACIÓN}

La educación es un proceso inherente al desarrollo histórico de toda sociedad. Su realización, su función y su finalidad están también ligadas a cada sociedad (Savoy, 1994; Bertrand et Valois, 1999). En ese sentido, todo concepto de educación está ligado a los intereses, aspiraciones y concepciones (Sarrasin, 1994), que cada sociedad desarrolla para una mejor integración de las nuevas generaciones, según las normas y reglas en ellas establecidas (Küper, 1993). La "educación es un proceso en el que los sujetos desarrollan sus potencias intelectuales mediante el uso de las estructuras públicas de conocimiento para construir una comprensión personal de las situaciones de la vida" (Elliott, 1994: 13). Dicho proceso incluye cuatro dimensiones de aprendizaje del patrimonio cultural: 1) el entrenamiento referido a la adquisición de las habilidades necesarias para el desarrollo de las actividades específicas que están en la base para comprender y desenvolverse en la realidad;2) la instrucción comprendida como un conjunto de procesos destinados a transmitir y retener las informaciones del medio social; 3) la iniciación referida a un conjunto de procesos para adquirir conocimientos, normas y valores socioculturales en la comunidad; y 4) la inducción como un proceso que permite al sujeto comprender los elementos de información (Stenhouse, 1998a; 1998b), como también las estructuras del conocimiento y pensamiento disponible y construido históricamente por la sociedad.

Manteniendo la misma lógica, la educación mapunche tradicional se define como:

“[...] una transmisión de la alta clase de la cultura mapuche; para preparar a un señor, un huerquen. El hijo de cacique necesitaba ser un buen orador, conocer la gente, saber sobre los secretos [...] saber de la historia, la tradición, las costumbres, la cultura, la justicia [...] conservar y defender la cultura. Ser capaz de decir al pueblo cuál es la tradición y la historia" (Bengoa, 1996:66).

La educación mapunche consiste en una enseñanza que favorece el ejercicio de la memoria, de la capacidad de describir y caracterizar los objetos y la situación de la vida social. El mapunzugun, en tanto lengua de los Mapunche, es un medio de adquisición, de retención, de descripción y de comunicación de saberes de la realidad empírica, necesarios para la comprehensión de la sociedad y la cultura, a través de la socialización.

\subsubsection{La SOCIALIZACióN}

La socialización es un proceso que permite la transmisión de la memoria histórica mapunche a través de la literatura oral, expresada por los miembros de la familia en el contexto de la comunidad (Carilao et al., 1998). Según Vásquez (1996), la socialización consiste en un proceso de integración de los sujetos en el marco de una cultura que cambia y que se transforma permanentemente, en vías de adquisición de saberes y del saber hacer que son necesarios en el contexto de la interacción social. De esta forma, la socialización permite la integración del sujeto a una colectividad que es influenciada y transformada por fuerzas externas e internas (Gohard-Radenkovic, 1999). En el mismo sentido, Rocher define la socialización como: 
“[...] el proceso por el cual la persona humana aprende e interioriza los elementos socioculturales en el transcurso de su vida, los integra a la estructura de su personalidad, bajo la influencia de las experiencias de relación con agentes sociales significativos, para adaptarse a su entorno social en cuyo seno debe vivir (Rocher, 1992:105, traducción libre).

Rocher precisa la definición en cuatro dimensiones de socialización: 1) los contenidos, 2) los mecanismos, 3) los agentes y 4) los medios. Los contenidos de socialización consisten en normas, saberes y reglas sociales necesarios para que los individuos puedan desenvolverse en el marco de su sociedad y cultura. Los mecanismos de socialización están referidos al aprendizaje y proceso de interiorización de los individuos sobre los contenidos sociales y culturales, para la formación de un estado de personalidad para actuar en la vida social y cultural. Es decir, la sociedad misma le asigna una obligación moral y ciertas normas, lo cual permite el control social como imperativo y como exigencia del medio social. Los agentes de socialización son las instituciones, los grupos generacionales y los movimientos sociopolíticos, económicos, educacionales y territoriales. En la primera categoría, el autor identifica la familia y la escuela como agentes socializadores institucionalizados, consciente o no, voluntario o no, su tarea es trasmitir el patrimonio sociocultural a las nuevas generaciones. Los medios de socialización están referidos a los medios de comunicación y las tecnologías modernas; diarios, periódicos, radio, la informática y medios de publicidad que transmiten fenómenos de masa e influyen los procesos de socialización en la colectividad.

En esa misma lógica, la educación transmite un conjunto de contenidos educativos consignados en el marco del currículum para formar al niño mapunche en el medio escolar y en el contexto de la comunidad.

\subsection{El Concepto de Currículum Escolar}

Denominamos currículum escolar al contenido cultural transmitido en el marco de la escuela, que es agente de segunda cultura para las nuevas generaciones. Hoy día, en el contexto general, existe un reconocimiento de que los padres como los "enseñantes" se preocupan de las necesidades educativas de los niños en relación con las necesidades de cada sociedad (Caouette, 1992:45). Según Gimeno (1995), el contenido del currículum escolar como prescripción y su aplicación conducen a un conjunto de experiencias formadoras, orientada hacia un aprendizaje en relación con lo deseado por cada sociedad. En consecuencia, el currículum es toda una intención de homogeneización de los ciudadanos a partir de un conjunto de contenidos socioculturales seleccionados en el marco del currículum prescrito y real. Esta intención, tal vez, puede ser consciente o no, pero es todo un proceso de transmisión ligado a una intención de instruir a las nuevas generaciones (Perrenoud, 1994b). El autor entrega una definición del currículum que considera tres dimensiones, las cuales están en una relación educativa: 1) el que dice relación con contenidos educativos programados, planificados: el "currículum formal"; 2 ) las experiencias vehiculizadas por la persona que aprende y que, en clases, se desarrolla en el contexto de las actividades propuestas por el enseñante (profesor) o una enseñante: es el "currículum real"; y 3 ) los aprendizajes que vienen a resultar en las actividades educativas: el "currículum oculto" 5 (Perrenoud, 1994b : 62).

De una forma más precisa, el currículum formal se relaciona con la estructuración de la experiencia de formación de los alumnos en

5 1) "celui de la "programmation" d'un parcours éducatif, notamment dans l'esprit de l'éducateur; C'est le niveau du curriculum rêve, prescrit ou formel, 2) celui des expériences qui vit l'apprenant et qui le transforment; C'est le niveau du curriculum réel ou réalisé, 3) celui des apprentissages qui en résultent" (Perrenoud, 1994b: 62). 
términos de finalidades y contenidos declarados y definidos desde afuera por el sistema educativo nacional que el educador de cada establecimiento debe aplicar. El contenido educativo definido en el currículum escolar está en relación con un proyecto de sociedad, una concepción determinada de vida y el desarrollo del ser humano en el marco de la organización escolar de cada establecimiento. En el marco del currículum real, las intencionalidades formales y ocultas no son necesariamente planificadas ni conscientes en tanto actividad educativa. Corresponde a lo que el docente realmente realiza en la práctica de enseñanza escolar, en el contexto particular de cada establecimiento: en la forma de concebir los contenidos disciplinarios, la negociación con los alumnos y los intereses y concepciones de la comunidad. En el marco del currículum oculto se genera un conjunto de experiencias coherentes de formación y de adaptación, como "un medio de vida sociocultural complejo, activo, donde se realizan negociaciones, interpretaciones e influyen valores, saberes y conocimientos" (Perrenoud, 1994a: 26; traducción libre).

En el contexto indígena, la incorporación de conocimientos al currículum formal se relaciona con un prestigio y un saber hacer de establecimientos escolares en la práctica de enseñanza escolar, en tanto mandato social (Montaluisa, 1988; Rockwell, 1995). En esta lógica, lo que se demanda formal o informalmente por las familias y comunidades indígenas, es una documentación de contenidos educativos transmitidos a las nuevas generaciones desde épocas pasadas, para ser incorporados a la educación del niño mapunche en el marco del currículum escolar. Sin embargo, el modo de vida en el contexto de la familia y la comunidad ha cambiado ¿Hoy día, cuáles son esos conocimientos y su proceso de transmisión? La perspectiva retenida para dar respuesta a esta pregunta es la exploración del concepto de representación social en relación con la educación y la transmisión del conocimiento sobre la relación de parentesco.

\subsection{La Representación Social de la Educación}

Antes de poner en relación la representación social y educación, es conveniente definir brevemente el concepto de representación social. Según Moscovici (1998), la representación social consiste en un proceso de fijación y objetivación de las ideas de los sujetos, para comprender los diferentes elementos de información del entorno social, cultural y natural. Así, todo objeto o comportamiento social es una realidad en sí y también una representación. Según Bronkart (1998), la representación de mundo reposa sobre unas unidades de representaciones delimitadas que se organizan en categorías relevantes en los sistemas de pensamientos y conocimientos socialmente compartidos. Desde esta perspectiva, las representaciones sociales colectivas "son producto de la cooperación que se extiende no sólo en el espacio sino en el tiempo (...); trasciende el ser individual y expresan una realidad más alta, de la propia sociedad" (Bonfil, 1993:168).

En el ámbito sociocultural, Moscovici (1993) indica que las ideas y el pensamiento de un grupo constituyen una representación colectiva que es consensual entre los miembros del grupo. Estos "pensamientos y estas ideas constituyen una representación social que marca las diferencias de un grupo en relación con la sociedad global" (Rouquette, 1998:15). De esta forma, la representación es una forma de actuar en respuesta a un consenso colectivo construido en la interacción social (Doise, 1993). Se puede suponer que en las comunidades mapunche rurales, los parientes poseen ideas definidas sobre los miembros de la comunidad que se ligan a un tronco parental común, en el espacio y el tiempo. Estas son representaciones que actúan como un saber para comprehender la realidad social y cultural.

La representación social de la educación está ligada a una idea de hombre y de sociedad (Savoy, 1994), objetivada en la estructura de pensamiento de la sociedad de pertenen- 
cia de cada sujeto (Moscovici, 1998). Para Bengoa (1996), en la familia mapunche, los contenidos de la educación son comunicados a través del discurso oral, lo cual expresa una concepción de educación que se relaciona con una idea de hombre y de sociedad mapunche. En este sentido, el individuo puede transformar, modificar y reproducir su representación a través de los discursos orales. En este proceso la valoración de la escuela y de la educación escolar están ligadas a una representación social (Montandon, 1994; Forquin, 1997), y particularmente en el contexto indígena en América Latina (Montaluisa, 1988). En consecuencia, la representación social de la educación es una estructura que es elaborada en forma permanente, a través de los discursos y de la literatura disponible en forma dialógica entre el pensamiento colectivo y el pensamiento individual retenido en la literatura (Moscovici, 1998; Gilly, 1993). En el marco del presente estudio la literatura se refiere al patrimonio cultural que se conserva en la memoria social de los individuos y se transmite oralmente como contenido educativo en el medio comunitario.

\subsection{La Relación de Parentesco como Contenido Educativo}

Una puesta en relación de los conceptos representación y educación nos permite comprender los procesos de transmisión del patrimonio cultural mapunche a los niños. En la lógica de representación del conocimiento sobre la relación de parentesco como contenido educativo, la memoria social constituye un aspecto pedagógico y didáctico a ser considerado desde un enfoque intercultural de la educación y un currículum escolar que atiende a la diversidad sociocultural.

En la perspectiva de Ouellet (1988) y Abdallah-Pretceille (1996), el enfoque intercultural de la educación no implica tanto la adquisición de conocimientos, saberes y categorías objetivas de la cultura del otro, sino más bien significa el descubrimiento del sujeto que aprende respecto de su propia subjetividad e identidad social y cultural. En el contexto latinoamericano, la interculturalidad en el ámbito educativo se entiende como un proceso "centrado en las relaciones diversas y múltiples de las culturas, donde la autonomía en las relaciones socioculturales esté orientada por principios como legitimidad de conocimiento, relatividad de las prácticas sociales y culturales, saberes y aspiraciones sociales" (Küper, 1993:111).

La relación de parentesco en el contexto de las familias y comunidades mapunche constituye una categoría de contenidos central en la transmisión de la memoria social a las nuevas generaciones. Aunque las familias y las comunidades han experimentado cambios y transformaciones, se continúa una transmisión de conocimientos sobre la relación de parentesco como un contenido para la formación de personas que respondan a una forma de ser mapunche. Ser mapunche significa la conformación de una personalidad de los individuos que se liga a un conocimiento del patrimonio sociocultural propio, para establecer relaciones sociales armónicas sobre la base de normas y valores establecidos en la comunidad. El resultado de este proceso educativo liga a las personas con ciertos intereses y finalidades educativas en relación al marco cultural mapunche y occidental en la sociedad chilena, lo cual constituye un objetivo inmediato de la educación intercultural.

\section{Método}

\section{Sujetos}

Se consideró una población de 18 personas de la comuna de Padre Las Casas, de la IX Región. Estas personas provienen de las comunidades Jewpeko, que significa lugar donde nacen las aguas, Kuzako, que significa lugar donde abunda el agua y piedras, y Chomiw, que significa lugar donde existen valles entre montañas. En Jewpeko han sido entrevistadas ocho personas, cuatro hombres y cuatro mujeres; en Kuzako han sido entrevistadas cinco personas, tres hom- 
bres y dos mujeres; y en Chomiw, han sido entrevistadas cinco personas, dos hombres y tres mujeres. En total se ha trabajado con 18 entrevistas: 11 padres de 25 a 50 años de edad y 7 kimche, personas sabias, mayores de 50 años de edad, quienes tienen una relación parental directa con niños que asisten a escuelas situadas en dichas comunidades. Las razones teóricas para trabajar con esta muestra son: 1) justificar que la representación del conocimiento sobre la relación de parentesco en la familia mapunche está validada por la memoria social de los kimche; 2) que los conocimientos de kimche permiten dar una mejor lectura sobre la representación de la relación de parentesco como contenido educativo en la familia mapunche; 3) obtener una descripción lo más justa posible del conocimiento mapunche sobre la relación de parentesco; 4) identificar y describir los contenidos educativos que subyacen a la relación de parentesco; e 5) identificar el punto de vista de miembros de la comunidad mapunche, respecto de aquellos contenidos deseables de ser integrados a la educación escolar en el Estudio y Comprensión del Medio Natural, Social y Cultural.

El análisis realizado en la investigación es de contenido, por unidades temáticas, en relación con las dimensiones e indicadores definidos en el modelo de análisis (Quintriqueo, 2002: 56). En este proceso, la codificación y categorización de las unidades de análisis han permitido organizar la información por temas y categorías (Gauthier, 1998). La unidad de análisis en la investigación corresponde a un contenido mínimo de la información expresada por las personas entrevistadas en relación con el objeto de investigación (Quivy, 1998). El análisis de contenido de la información ha sido realizado a la luz de la pregunta y objetivo de investigación. La recolección y análisis de datos ha sido efectuada entre enero y febrero de 2002 (Quintriqueo, 2002: 57).

\section{Resultados}

\section{La Relación de Parentesco como Contenido del Currículum Escolar}

La relación de parentesco consiste en un conjunto de conocimientos construidos en la interacción social entre los miembros del tronco parental común, en una relación dialógica con los contenidos retenidos en la memoria social mapunche. La organización de estos contenidos sobre la relación de parentesco está dada sobre la base de un modelo y mecanismo de socialización en el contexto de la familia y la comunidad.

\subsection{Los Contenidos Relativos a la Relación de Parentesco y su Proceso de Socialización}

Los kimche (personas sabias) son quienes poseen el conocimiento sobre la relación de parentesco adquirido en su proceso de socialización y conservados en categorías relevantes de la memoria social sobre el reñma, tuwün y küpan [características sociales, económicas, culturales y políticas de las personas]. En este proceso se observan agentes, mecanismos, espacios, prácticas y contenidos de socialización.

Los primeros agentes de socialización que transmiten la relación de parentesco son los padres de familia mapunche. Los conocimientos que los niños aprenden acerca del parentesco son adquiridos a través de la relación de diálogo con sus padres y pares parientes, ya sea en la familia, en la comunidad o en el medio escolar.

Los principales mecanismos de socialización de contenidos sobre la relación de parentesco hoy día en la familia mapunche son el nüxam, que significa la conversación; el gübam, que significa un modo propiamente mapunche de organizar y transmitir el Conocimiento sobre la relación de parentesco; y el kimeltuwün, que significan el aprendizaje-enseñanza del kimün (conocimiento) referido al parentesco. En forma más preci- 
sa, el nüxam se concibe como una forma de comunicar conocimientos construidos por los ancestros en la familia y la comunidad, expresados en mapunzugun -lengua mapunche-. En cambio, en aquellas personas que no hablan el mapunzugun, el nüxam como mecanismo de transmisión de contenidos se realiza en castellano. En tanto, el epewkantun (jugar a representar la realidad) y el piam (decir, relato) representan y organizan situaciones vividas por los antepasados y que han sido retenidas en la memoria social, expresadas a través del discurso oral en mapunzugun. El wewpin (oratoria, discusión) presenta una estructuración lógica del conocimiento sobre la relación de parentesco en la comunidad y la proyección de ésta, a través de su transmisión a las nuevas generaciones.

El gijatuwe como espacio de socialización existente en el contexto de la familia y el uso del mapunzugun, constituyen mecanismos importantes de representación del valor de la relación de parentesco en la comunidad mapunche. El objetivo principal es poner en escenario público aquello que los kimche saben respecto de la memoria social, para ser transmitido a los niños y los miembros de la comunidad en general. En este proceso, se identifican principios básicos para la comunicación de contenidos con relación al parentesco. Por ejemplo, kimelniel fey ta kimi significa a quien se enseña es quien aprende; kimalu ta kimkey significa aquel que quiere aprender aprende; e inatuwlu ta kimkey significa el que sigue la memoria de sus antepasados es quien tiene conocimiento y saber sobre la experiencia personal y social con relación al parentesco en espacios de socialización formal e informal. Uno de estos espacios es el gijatuwe, lugar de encuentro donde las personas participan en el gijatun (rogativa) y están llamadas a escuchar los contenidos referidos al conocimiento sobre el parentesco, a través del discurso oral en mapunzugun: el nüxam, el gübam, el gijatun.

Las prácticas de transmisión de conocimientos sobre la relación de parentesco en la comunidad mapunche son: el palin, gijatun, gübam, nüxam y eperwkantun. El palin es entendido como una actividad deportiva propia, la cual se concibe como una instancia de encuentro entre parientes, para fortalecer ideas y pensamientos que han sido construidos en el pasado cultural y social con relación al conocimiento sobre el parentesco en la comunidad. El gijatun consiste en un discurso dialógico, formal y situacional que busca ligar a las personas con su pasado social y cultural, desde una visión religiosa mapunche para orientar la relación de los sujetos con su tronco parental y Günechen Ser Superior-. El gijatun es un relato dirigido a niños y jóvenes, con el fin de que éstos escuchen contenidos sobre la relación de parentesco y asumen una proyección de dichos conocimientos en las nuevas generaciones. El gübam se entiende como un relato que constituye un método para organizar los contenidos a comunicar y con propósitos más o menos definidos. Por ejemplo, formar una persona para aprender a respetar, para tratar a las personas en el cariño, en la confianza y entregar conocimientos de cómo son los mapunche y la historia acerca de los parientes en la comunidad (Quintriqueo, 2002). El gübam busca explicitar en forma organizada los conocimientos a ser enseñados y expresa el objetivo que se propone para la enseñanza.

El kimeltuwün se entiende como un proceso de aprendizaje y enseñanza que ocurre entre dos personas que tienen intención de conocer el parentesco y ser formadas en contenidos referidos a la relación de parentesco, en el contexto de la familia y la comunidad mapunche. El momento para la transmisión de contenidos sobre la relación de parentesco se relaciona con experiencias de prácticas socioculturales formales y colectivas entre padres, hijos y miembros de la comunidad; el pentukun significa saber sobre la situación social, económica, política y cultural que vive el parentesco. En esa misma lógica, el pentukuwün, es un saludo formal mapunche y como tal, una de las prácticas más complejas y organizadas utilizadas en el gijatun, 
para "expresar lo que se siente y lo que se sabe" sobre la relación de parentesco. En la realidad implica una actuación de cortesía de los individuos en la vida cotidiana y formal, personal y colectiva entre las personas de la familia y la comunidad, lo cual es deseable que sea enseñado como contenido en la socialización, a través del gübam. Los contenidos transmitidos a través de la socialización se ligan a un pasado cultural de los grupos parentales y el conocimiento respecto a la experiencia de relación de parentesco entre los miembros de la comunidad en la actualidad.

Los contenidos conceptuales sobre la relación de parentesco se expresan en una lógica de pensamiento sobre el tuwïn (ascendencia materna), el küpan, (ascendencia paterna) y el reñma, (red de parentesco) que se ha construido como producto de la ramificación del tronco parental común en la actual comunidad mapunche. Este conocimiento incluye igualmente las prácticas de relaciones sociales y culturales con los miembros del parentesco en la familia. Por ejemplo, el zuguwün significa dialogar entre parientes y miembros de la comunidad; wixankontuwün significa visitarse entre miembros del parentesco y otras personas de la comunidad. Así, el wixankontuwiün y el wixangen responden a una intención planificada del núcleo familiar para reconocerse como miembros de un parentesco. Estas visitas se organizan en base a un contenido, una situación y un propósito bien definido: a) los contenidos se refieren al tuwün y al küpan; b) la situación corresponde al cambio permanente que experimentan las familias con relación al conocimiento sobre el tronco parental; y c) el propósito se relaciona con hacer comprender a los individuos sobre su pertenencia a un tronco parental, es decir, aprender a ser mapunche. El mogeyewün significa practicar la visita entre los parientes. Los contenidos sobre la relación de parentesco se relacionan también con el rakizuam, que significa la lógica de pensamiento propio del mapunche; el mapunche kimün, que significa los saberes y conocimientos propios del mapunche; y el inatuwün, que significa indagar en la memoria social sobre la relación de parentesco.

Los contenidos valóricos se expresan a través del yamün, que significa la relación de cortesía, una valoración e identificación de los niños con los miembros del parentesco. La forma de saludar a las personas de la comunidad adquiere importancia en la familia, para desarrollar sistemas de valores en torno al conocimiento sobre la relación de parentesco. Así, el "cariño" que los adultos y kimche del tronco parental demuestran a los niños en las prácticas de relaciones sociales, se observa como principal variable para que éstos desarrollen una motivación por tener un conocimiento sobre el parentesco. Éste es un conocimiento expresado en la demostración de valor, saber y actitud de reconocimiento que asumen los niños en la relación cotidiana y formal con los miembros del tronco parental en la comunidad, lo cual permite que los jóvenes reconozcan el origen de sus parientes en lugares públicos. Por ejemplo, reconocer al pariente en la calle, en el micro bus que tiene recorrido por la comunidad y en relaciones sociales espontáneas que ocurren en la ciudad.

En la Tabla $\mathrm{N}^{\mathrm{o}} 1$ se observa que existe un patrimonio de conocimientos sobre la relación de parentesco retenido en la memoria social y que se transmite en el contexto familiar ¿Será posible que este contenido educativo pueda ser contextualizado y formalizado en la perspectiva de una eventual incorporación al currículum escolar, en el marco del subsector de aprendizaje Estudio y Comprensión del Medio Natural, Social y Cultural? La pregunta se plantea en razón de las transformaciones vehiculizadas en los procesos de transmisión de los contenidos descritos en el punto siguiente. 


\subsection{Conocimientos Sobre la Relación de} Parentesco como Contenido Educativo Escolar

La memoria social mapunche hace referencia a la relación de parentesco del pasado y permite identificar algunos contenidos y principios específicos para la formación del niño mapunche en el contexto escolar: 1) kümeyawal ta che, consiste en la "actitud" de comportarse con rectitud en la relación con las personas de un mismo tronco parental; 2) yamüwal ta che, significa "una relación de estima, respeto y conocimiento" entre personas de un mismo tronco parental en la comunidad; 3) küme rakizuam ta niael, consiste en "formar a la persona para que asuma un buen tipo de pensamiento" requerido en su familia y la comunidad mapunche ac- tual; 4) küme che geal, significa una persona que busque ser modelo, "ser solidario" dentro del grupo parental; 5) kim che geal, una acción educativa que tenga por objeto "la formación de una persona mapunche basada en el conocimiento"; 6) küme inayal ta ñi zugu, una persona que busque "seguir el buen actuar, ser firme en una forma de pensar"; 7) ajkütual ta zugu, significa "formar una persona capaz de escuchar los saberes y conocimientos", sean mapunche o no; y 8) kümeke gübam ta yenial, "ser una persona portadora del buen consejo" sobre la memoria social. En esta perspectiva, transmitir una forma de ser mapunche, significa un proceso de construcción de identidad individual y social con relación al conocimiento sobre la relación de parentesco en la comunidad.

Tabla N¹. La Relación de Parentesco como Conteni do Educativo Mapunche

\begin{tabular}{|c|c|c|c|}
\hline $\begin{array}{l}\text { Dimensiones de } \\
\text { socialización }\end{array}$ & Contenidos educativos & $\begin{array}{l}\text { Principios de } \\
\text { socialización }\end{array}$ & Finalidades educativas \\
\hline Agentes & $\begin{array}{l}\text { Kimche, padres, } \\
\text { miembros del } \\
\text { parentesco; familia- } \\
\text { Comunidad }\end{array}$ & \multirow[t]{2}{*}{ Kimalu fey ta kimi } & $\begin{array}{l}\rightarrow \text { Conservar y retransmitir el } \\
\text { saber y conocimiento } \\
\text { educativo sobre la relación de } \\
\text { parentesco }\end{array}$ \\
\hline Mecanismos & $\begin{array}{l}\text { Gübam, Nûxam, } \\
\text { Kimeltuwūn, wewpin }\end{array}$ & & $\rightarrow \begin{array}{l}\text { Transmitir el conocimiento } \\
\text { educativo }\end{array}$ \\
\hline Espacios & $\begin{array}{l}\text { Paliwe, Gijatuwe, } \\
\text { familia, comunidad }\end{array}$ & Kimlu ta kimkey & $\begin{array}{l}\rightarrow \text { Dar lugar al encuentro entre } \\
\text { miembros del parentesco en la } \\
\text { familia y la comunidad }\end{array}$ \\
\hline Prácticas & $\begin{array}{l}\text { Palin, Gijatun, Pentukun, } \\
\text { Gübamtun, Wixangen, } \\
\text { Kimeltun }\end{array}$ & $\begin{array}{l}\text { Familia ta ti inchin } \\
\text { Inatuwlu ta } \\
\text { kimkey }\end{array}$ & $\begin{array}{l}\text { Aplicar el conocimiento } \\
\text { educativo en la vida social y } \\
\text { cultural }\end{array}$ \\
\hline $\begin{array}{l}\text { Contenidos } \\
\text { th Valóricos }\end{array}$ & $\begin{array}{l}\text { Küme rakizaum } \\
\text { Küme kimün } \\
\text { Yamün } \\
\text { Küme chegen }\end{array}$ & \multirow{2}{*}{$\begin{array}{l}\text { Kiñe mojfüñ ta } \\
\text { mapunche }\end{array}$} & $\begin{array}{l}\rightarrow \text { Formar el az, es decir, la } \\
\text { personalidad del niño } \\
\text { mapunche }\end{array}$ \\
\hline to Conceptuales & $\begin{array}{l}\text { Rakizuam, Kimün, } \\
\text { Ren̂̉ma, Tuwün, Küpan }\end{array}$ & & $\begin{array}{l}\rightarrow \text { Formar una lógica de } \\
\text { pensamiento propio }\end{array}$ \\
\hline \multirow{2}{*}{ t5 Actitudinales } & & \multirow{2}{*}{$\begin{array}{l}\text { Zapigekey ta pu } \\
\text { xemüm }\end{array}$} & $\begin{array}{l}\rightarrow \text { Formar modelos de persona } \\
\text { mapunche }\end{array}$ \\
\hline & $\begin{array}{l}\text { Wixankontưwùn, } \\
\text { Zuguwûn, Mogeyewûn }\end{array}$ & & $\begin{array}{l}\rightarrow \text { Tener conocimientos sobre la } \\
\text { relación de parentesco }\end{array}$ \\
\hline
\end{tabular}


El conjunto de contenidos y principios que aquí identificamos, en relación con las categorías de conocimientos sobre la relación de parentesco deseables de transmitir a las nuevas generaciones, constituyen aspectos importantes en el propósito de auto-identificación y reidentificación del niño mapunche. La reidentificación es entendida como un aprendizaje de la pertenencia cultural, social y étnica, a través de procesos de escolarización que considere el patrimonio cultural mapunche. Los antecedentes aportados por la investigación ponen en evidencia que los padres y miembros de la comunidad desean que la escuela asuma una parte de la responsabilidad en la transmisión de contenidos educativos basados en el conocimiento propio del niño mapunche en el medio escolar. En la hipótesis planteada, encontramos legítimo y pertinente una enseñanza escolar que tenga por objeto la formación de educandos identificados con su cultura y sociedad. Según este razonamiento, las actividades de formación inicial en Educación Preescolar y Educación Básica Intercultural en relación con el Estudio y Comprensión del Medio Social y Cultural, es pertinente la incorporación de contenidos educativos asumiendo las lógicas y significados del conocimiento propio de la familia y la comunidad mapunche. En esa lógica, debiera existir el espacio para una formación que permita al futuro profesor desarrollar competencias en la transmisión de contenidos en referencia a la memoria social mapunche en contexto intercultural. En el presente artículo, se define el contexto intercultural como un conjunto de circunstancia y hechos que adquieren valor y significado en la relación de sujetos mapunche y no mapunche en el contexto regional y nacional.

\section{Discusión}

La memoria social mapunche se desarrolla en relación con el conocimiento referido a la relación de parentesco, en tanto representación construida sobre la base de la experiencia de interacción social entre los miem- bros del tronco parental común. Este es un proceso de interacción social que, consciente o inconscientemente, tiene como propósito transmitir contenidos sobre la relación de parentesco y reconocer a los miembros del tronco parental que comparten el medio social, cultural y natural en la comunidad. En esa perspectiva, la memoria social mapunche sobre la relación de parentesco constituye una representación que se construye en un proceso de interacción social e histórico (Montaluisa, 1988; Bronkart, 1998; Moscovici, 1998), lo cual se expresa en el constante recuerdo sobre la experiencia de prácticas sociales y culturales con los miembros del tronco parental o con personas de otras comunidades. En la perspectiva de Moscovici (1993), esta es una experiencia de interacción social colectiva que constituye todo un consenso entre los miembros de un grupo social, respecto a pensamientos e ideas en relación con la sociedad y que se transforma a través del discurso; sea oral o escrito.

La representación del conocimiento sobre la relación de parentesco como contenido educativo responde a una construcción personal, consensual y colectiva, en base a prácticas sociales y culturales que van dando estructura tanto al contenido como a su proceso de transmisión a las nuevas generaciones. En la perspectiva de Bronkart (1998) y Doise (1993), esta es una forma de representación que es formal, elaborada y legitimada en la reflexión colectiva. Desde el pasado cultural mapunche, las prácticas sociales y culturales como el gijatun, el pentukun y el wixankontuwïn, son estructurados y representados en el colectivo social y legitimados formalmente a través de consensos. Estas representaciones formales constituyen conocimientos sobre la relación de parentesco en la familia y la comunidad mapunche, expresados en el saber, valor, respeto y concepciones que estructuran categorías de contenidos educativos deseables de transmitir a las nuevas generaciones. 
A pesar de las transformaciones sociales de la familia y la comunidad mapunche, estos conocimientos con relación al parentesco aún existen y se continúan transmitiendo en el contexto de la socialización familiar con relación a tres categorías conceptuales identificadas más arriba: el küpan, el tuwüin y el reñma. En la investigación se constata que la relación de parentesco que asume la familia hoy, no se da sólo entre mapunche, sino también con personas no mapunche que se han integrado al grupo parental, en el contexto de la familia y la comunidad. Además, es posible encontrar elementos de la tecnología y la comunicación, el trabajo al estilo occidental y la experiencia de escolarización que de algún modo están influyendo la existencia o no de las prácticas de relación de parentesco en el contexto de la familia. En esta perspectiva, la escolarización se constata como un ámbito de socialización y de influencia sobre la relación de parentesco en la familia mapunche actual. Por una parte, la escolarización es un fenómeno que se expresa en dos puntos de vistas divergentes entre sí. 1) los kimche señalan que es un proceso donde los niños generan un distanciamiento progresivo respecto del conocimiento sobre la relación de parentesco en la familia y la comunidad; y 2) los padres jóvenes señalan que la escuela constituye un espacio de socialización entre niños que pertenecen a un mismo tronco parental. Por otra parte, existe convergencia entre kimche y padres jóvenes en dos aspectos: 1) todos los entrevistados en la investigación indican una ausencia progresiva de contenidos sobre la relación de parentesco mapunche en la socialización familiar; y 2) los conocimientos sobre la relación de parentesco están en los kimche, quienes han conservado la memoria social mapunche en la comunidad.

La memoria social mapunche con relación al parentesco está organizada en la literatura oral y con relación al pasado cultural para dar lectura a la realidad social y cultural actual. En este caso, la literatura oral se refiere al saber organizado en marcos conceptuales propios del mapunzugun, lo cual permite la retención de las nociones de tiempo-espacio y contenidos sobre la relación de parentesco. La noción de tiempo está ligada fundamentalmente al -kuyfi-, que significa el tiempo transcurrido, fantepu, que significa actualmente y kiñen antü, que significa el tiempo que viene. Estas son tres dimensiones del tiempo y espacio en el cual las personas entrevistadas han situado sus discursos para expresar la situación del conocimiento sobre la relación de parentesco deseables de transmitir a niños de edad escolar.

La dimensión tiempo-espacio, se relaciona también con el recorrido sobre la relación de parentesco que han vivido los individuos en el contexto de la familia y la comunidad mapunche rural. Y el medio se relaciona con los elementos y características del paisaje de la comunidad, en cuya relación se expresa un conocimiento y valor de la relación de parentesco. Hoy día, los espacios y prácticas socioculturales situacionales en el contexto de la familia, aún constituyen oportunidades de socialización, consciente o no, es flexible, organizado, abierto y bidireccional (Rocher, 1992; Vásquez y Martínez, 1996), donde necesariamente aprenden todos los actores del proceso educativo.

En esa lógica, se observa que existe un modelo de socialización y contenidos sobre la relación de parentesco que las familias mapunche desean que sean integrados como contenidos del currículum escolar intercultural y en las actividades educativas escolares. En el nivel de establecimiento escolar, esto puede constituir un Proyecto Educativo Institucional, donde los padres y miembros de la comunidad asumen una responsabilidad en la definición de contenidos y finalidades educativas. Ya existen en otros contextos, proyectos de desarrollo y de investigación de esta naturaleza en comunidades indígenas, en Canadá (Vick-Wesgate, A., 2002; Da Silveira, Y., Maheux, G., Simard, D. et Quilaqueo, D., 2002; Bergeron, C., Maheux 
G., 1999; Maheux, G., 1996, 2001) y en Australia (Harris, S., 1990).

Las comunidades Inuit ${ }^{6}$ en Québec-Canadá, por hablar sólo de ésta, considerando el tiempo y la energía invertida para construir un currículum intercultural y bicultural, después de treinta años, han asumido progresivamente la responsabilidad frente a la educación escolar de las nuevas generaciones. Estamos seguros, el desarrollo del aspecto cultural indígena en la escolarización evoluciona lentamente con respecto al occidental. Si nos referimos a Harris (1990), la consideración de la responsabilidad de la comunidad local en la escuela es un proceso de apropiación y de incorporación sociocultural que avanza lentamente en razón, netamente, de las diferencias existente entre la cultura indígena y la cultura occidental. En este proceso, la institución educativa es como en otras partes, la más pura manifestación de la diferencia entre la cultura indígena y occidental. Sin embargo, para que estas comunidades inuit obtengan, según la ley, la responsabilidad de gestionar los servicios administrativos de la educación para la población que reside sobre su propio territorio, participan en la generación de propuestas educativas propias. Es así como ellos sostienen después de treinta años, el desarrollo de un proyecto integrado a la formación de profesores de origen Inuit y del currículum escolar del establecimiento en inuktitud (lengua hablada por la población Inuit) y una lengua segunda, francés o inglés, según la elección de los padres. Sin embargo, ello se relaciona con una situación educativa compleja: el desafío consiste en desarrollar un proyecto de educación, un contenido educativo que permitirá a los jóvenes mantener su identidad cultural, todo lo cual está relacionado con la adquisición de un nivel de escolaridad adecuado para insertarse socialmente y profesionalmente en la sociedad actual. La principal apuesta es asegurar la aprobación escolar del conjunto de la población de las comunidades inuit y otras comunidades indígenas.

El contexto de administración de la educación ofrecida a la población de las comunidades mapunche es un poco diferente: la apertura al proceso de cambio en la gestión de Proyectos Educativos Institucionales y Proyectos Curriculares para cada establecimiento escolar que descanse sobre la comunidad local existe legalmente sólo desde 1996. Por lo tanto, existen iniciativas recientes en comunidades mapunche que tienen como propósito considerar la responsabilidad de la comunidad en los Proyectos Educativos Institucionales. Una acción posible de proponer es avanzar en la construcción de un cuerpo de conocimientos que tenga por objeto responder a preguntas claves en relación a la formación de persona en contextos interculturales ¿Qué aspectos y lógicas del conocimiento mapunche considerar para el diseño de un modelo pedagógico y didáctico para la Formación Inicial Docente en Educación Intercultural, para mejorar la calidad de la educación y la participación social mapunche, a través de la educación escolar? ¿Cuáles son los aspectos legales y curriculares que permitirían la organización de ciertas categorías de contenidos y finalidades educativas pertinentes para la formación de persona en el medio escolar con características interculturales? ¿Existe una intención de las comunidades mapunche de avanzar en un trabajo colaborativo en el diseño de proyectos educativos y en el desarrollo de investigaciones que tengan por objeto la sistematizacción de contenidos y finalidades educativas propias, para mejorar la calidad y equidad de la educación?

6 El pueblo indígena Inuit habita el territorio que se ubica al Norte de la Provincia de Québec. Canadá. 


\section{Referencias}

Abdallah-Prestceille, Martine. (1996). Vers une pédagogie interculturelle. Paris: Anthropo, $222 \mathrm{p}$.

Bengoa, José. (1996). Historia del Pueblo Mapuche. 5e Edición. Santiago de Chile: Ediciones Sur Colección Estudios Históricos, $485 \mathrm{p}$.

Bergeron, Charles, Maheux, Gisèle. (1999). Le difficile défi de la scolarisation des Inuit $d u$ Québec, Université du Québec en AbitibiTémiscamingue, Rouyn-Noranda, p.7.

Bertrand, Yves y Valois, Paul. (1999). Fondements de l'éducation pour une nouvelle société. Montréal: Éditions nouvelles, 297 p.

BONFIL, GuILlERMO (1993). Implicaciones éticas de sistemas de control cultural. En OLIVÉ, L. Ética y diversidad cultural. Fondo de cultura Económica, México.

Bronckart, JeAn-PAUL. (1998). “Langage et représentaion". En Sciences Humaines, no 21 , p. 20-23

Caouette, Charles. (1992). Si on parlait d'éducation: pour un nouveau projet de société. Montréal: VLB Éditeur, 262 p.

Carilao, Domingo, Garnica Iván, HuenchuLAF Rosa Y LLANQUINAO GABRIEL. (1998). La socialización del niño Mapunche, en el ámbito de la familia, el lof-che y la escuela. Tesis para optar al grado de Licenciado en Educación. Universidad Católica de Temuco. Temuco, $134 \mathrm{p}$.

Chile, Ministerio de Educación. (1990/1998). Ley Orgánica Constitucional de Enseñanza. Santiago de Chile, 68 p.

Chile, Ministerio de Educación, Consejo SuPERIOR DE EDUCACIÓN. (1999). Decreto supremo número 240. Objetivos Fundamentales y Contenidos Minimos Obligatorios de la Educación Chilena. Santiago de Chile, 136 p.
Chile, Ministerio de Educación. (1996). Plan y Programa de Estudio para el Primer y Segundo Año de Enseñanza Básica (Nivel Básico 1). Santiago de Chile, $241 \mathrm{p}$.

Chile, Ministerio de Educación. (1997). Plan y Programa de Estudio para el Primer y Segundo Año de Enseñanza Básica (Nivel Básico 2). Santiago de Chile, $327 \mathrm{p}$.

Da Silveira, y. Maheux, G. Simard, D. ET Quilaqueo, D. (2002). Construction de savoirs professionnels et échanges interculturels en contexte de formation des enseignant-e-s inuit et mapunche. Collectif interculturel. Vol V, no 2, IRFIQ, p. 167-185.

Diaz, Francisco. (1997). La escuela Multicultural: principales retos. En PUBLICACIONES, ESCUELA UNIVERSITARIA DEL PROFESORADO DE EGB. no 25-26-27, p. 185-195.

DOISE, WILLEM. (1993). “Attitudes et représentations sociales". En Des représentations sociales, sous la dir. de Denise Jodelet. Paris: Presses Universitaires de France, p. 220 - 237.

ELLIOTT, John. (1994). La Investigación-acción en educación. $2^{\mathrm{e}}$ Edición. Madrid: Ediciones Morata, $235 \mathrm{p}$.

Essomba, Miquel. (1999). Construir la Escuela Intercultural: reflexiones y respuestas para trabajar la diversidad étnica y cultural. Barcelona: Graó Editorial, 196p.

FARON, Louis. (1997). Antupainamko; Moral y Ritual Mapuche. Santiago de Chile: Ediciones Mundo, $200 \mathrm{p}$.

FoERSTER, ROLF. (1988). Organizaciones, lideres y contiendas mapuche (1900-1970). Santiago: Ediciones Mundo, 430 p.

ForQuin, J.-Claude. (1997). Les sociologues de l'éducation américains et britanniques. Présentation et choix de textes. Bruxelles: De Boeck Université, 390 p. 
Gauthier, Benoît. (1998). Recherche sociale. De la problématique à la collecte des données. Ste-Foy: Presses de l'Université du Québec, 529 p.

Gilly, Michel. (1993). "Les représentations sociales dans le champ éducatif". En Des représentations sociales, sous la dir. de Denise Jodelet. Paris: Presses Universitaires de France, p. 363 - 386.

Gimeno, José. (1995). El currículum: una reflexión sobre la practica. Madrid: Ediciones Morata, $423 \mathrm{p}$.

Gissi, Nicolás. (1997). Aproximación al conocimiento de la memoria Mapuche-huilliche en San Juan de la Costa. Tesis para optar al título de Antropología Social. Universidad de Chile, Facultad de Ciencias Sociales, Santiago de Chile, $138 \mathrm{p}$.

Gohard-Randenkovic, A. (1999). Communiquer en langue étrangère. New York: Peter Lang S.A., 249 p.

Guevara, Tomas. (1904). Costumbres Judiciales; enseñanza de Los Araucanos. Santiago de Chile: Capítulo no 1, Imprenta Cervantes, p. 1-85.

HARRIS S. (1990). Two-Way Aboriginal Schooling Education and Cultural Survival. Canberra, Australia. Aboriginal Studies Press.176p.

KüPer Wolfgang, (1993). Pedagogía Intercultural Bilingüe. Fundamentos de la educación bilingüe. Quito: ABYA-YALA.

Maheux, G. (2001). Le développement professionnel et institutionnel de l'éducation en milieu inuit: analyse d'une pratique de construction de savoirs en contexte biculturel. La recherche en éducation et le développement de la pratique professionnelle en enseignement. (Éd.) André Beauchesne, Stéphane Martineau et Marc Tardif, Actes du $13^{e}$ congrès de l'AMSE, Éditions du CRP.

Maheux, G., Kenuayak, A., Simard, D., Paradis, V.Et Al. (1996). Stratégie de collaboration au développement de l'école communautaire en milieu inuit: Le pr ojet des écoles de Puvirnituq et d'Ivujivik, 64 $4^{\text {ième }}$ Congrès de l'ACFAS, Université McGill, 29p.

Merino, María y Quilaqueo, Daniel. (2003). Ethnic Prejudice Against the Mapuche in Chilian Society as a Reflection of the Racist Ideology of the Spanish Conquistadors. American Indian Culture and Research Journal 27:4, 105-116.

Montaluisa, Luis. (1988). Comunidad, escuela y currículo. CONAIE. Confederación de $\mathrm{Na}$ cionalidades Indígenas. Santiago de Chile: Orealc, 235 p.

Montandon, Cléopâtre. (1994). "L'articulation entre les familles et l'école: sens commun et regard sociologique". In L'éducation prisonnière de la forme scolaire?, sous la dir. de Guy Vincent. Lyon: Presses Universitaires de Lyon. p. 149-171.

Moscovici, Serge. (1993). “Des représentations collectives aux représentations sociales: éléments pour une histoire". En Des représentations sociales, sous la dir. de Denise Jodelet. Paris: Presses Universitaires de France, p. 62-86.

(1998). “Comment voit-on le monde?: représentations sociales et réalité". In Revue Sciences Humaines no 21, p. 20-23.

Noggler, Albert. (1972). Cuatrocientos años de Misión entre los Araucanos. Padre las Casas, Temuco: Editorial San Francisco, 485 p.

Ouellet, Fernand (dir.). (1988). Pluralisme et école. Québec: Institut québécois de la recherche sur la culture, $593 \mathrm{p}$.

Perrenoud, Philippe. (1994a). Métier d'élève et sens $d u$ travail scolaire. Paris: ESF édition, $207 \mathrm{p}$.

(1994b). “Curriculum: le formel, le réel, le caché". In. La pédagogie: une encyclopédie pour aujourd'hui. Paris: ESF édition, p. 61-76. 
Quilaqueo, Daniel y Quintriqueo, Segundo. (2003). "Prejuicio étnico subyacente hacia lo mapunche en el proyecto de formación inicial docente en educación intercultural bilingüe". Ponencia presentada al $51^{\circ}$ Congreso Internacional de Americanistas. Simposio MES - 7. "Políticas lingüísticas y proyectos de educación intercultural: Una visión desde los movimientos y comunidades indígenas en América". Mesa 5: Política intercultural y formación de profesores indígenas. Universidad de Chile. Santiago de Chile.

Quilaqueo, Daniel y Merino María. (2003). Estereotipos y prejuicios étnicos hacia los mapuches en textos complementarios a la asignatura de Historia. Campo Abierto, $\mathrm{N}^{\circ} 23$, pp. 119-135.

Quintriqueo, Segundo, Ancavil Brigida, Catrilaf Norma, Curihuinca Carmen y Paillan ERnesto. (1996). Procesos de construcción de conocimientos mapunche en relación a los wigkul y su socialización en la escuela-comunidad. Tesis para optar al grado de Licenciado en Educación. Universidad Católica de Temuco, Temuco, 99 p.

QuintriqueO, S. (2002). "Representación de la relación de parentesco que la familia mapunche posee y su transmisión a niños mapunche de edad escolar primaria en relación con los contenidos de Formación Inicial Docen$t e^{\prime \prime}$. Tesis de Maestría en Educación realizado “À l'Université du Québec en Abitibi-Temiscamingue, au QuébecCanada", de 1999 a 2002.

Quivy, Raymond Y CAmpenhoudt, L. Van. (1998). Manual de Investigación en Ciencias Sociales. México: Edición Limusa, 269 p.
ROCHER, GuY. (1992). Introduction à la sociologie générale: l'action sociale, l'organisation sociale, le changement social. Québec: Éditions Hurtubise, 136 p.

RocKWELL, ElsIE. (1995). La escuela cotidiana. México: Fondo de Cultura Económica, 238 p.

RouquetTe, Michel-L. (1998). Introduction à l'étude des représentations sociales. Grenoble: Presses Universitaires de Grenoble, 159 p.

SARrAsin, Robert. (1994). "Bilinguisme et biculturalisme chez les Atikamekw". En Revue canadienne de l'éducation. Vol. 19, no 2, p. 165-181.

SAVOY, U.v. FÉLIX. (1994). Educación y Formación Humana. Buenos Aires: Hvmanitas, 325p.

Stenhouse, LaWrence. (1998a). La Investigación como base de la Enseñanza, $5^{\circ}$ edición. Madrid: Ediciones Morata, 138 p.

(1998b). Investigación y desarrollo del currículum, $4^{\circ}$ edición. Madrid: Ediciones Morata, 319 p.

VÁsquez, ANA y MARTíneZ, IsABEl. (1996). La socialisation à l'école: approche ethnographique. Paris: Presses Universitaires de France, $223 \mathrm{p}$.

Vick-Wesgate, AnN.(2002). Nunavik InuitControlled Education in Arctic Quebec. Calgary: University of Calgary Press, 416p. 
